\title{
When Poisons Cure: The Case of Arsenic in Acute Promyelocytic Leukemia
}

\author{
Carmelo Gurnari $^{\mathrm{a}} \quad$ Eleonora De Bellis ${ }^{\mathrm{a}}$ Mariadomenica Divona ${ }^{\mathrm{a}}$ \\ Tiziana Ottone $^{a}$ Serena Lavorgna ${ }^{a}$ Maria Teresa Voso ${ }^{a, b}$ \\ aDepartment of Biomedicine and Prevention, University of Rome Tor Vergata, Rome, Italy; \\ ${ }^{b}$ Fondazione Santa Lucia, Laboratorio di Neuro-Oncoematologia, Roma, Italy
}

\section{Keywords}

Arsenic · Acute promyelocytic leukemia - Antitumor activity · All-trans-retinoic acid · Targeted therapy

\begin{abstract}
Arsenic has been known for centuries for its double-edged potential: a poison and at the same time a therapeutic agent. The name "arsenikon," meaning "potent," speaks itself for the pharmaceutical properties of this compound, questioned and analyzed for at least 2000 years. In the last decades, acute promyelocytic leukemia (APL) has evolved from a highly fatal to a curable disease, due to the use of all-transretinoic acid and, more recently, arsenic trioxide combinations. The success of these entirely chemo-free regimens increased the awareness of APL and reduced the prevalence of early deaths, which was an impending issue in this disease. Further improvements are expected with the next use of oral arsenic formulations, which will allow a complete outpatient approach, at least in the post-induction settings, further improving patients' quality of life. The wide use of standardized approaches in APL will also help unravel long-standing open questions, including the pathogenesis, prevention, and treatment of the differentiation syndrome and of short-term organ toxicities. In the long term, the study of survivorship issues, such as fertility and organ-related and psychological damages, in the increasing number of survivors will help further improve their life after APL.

๑) 2020 S. Karger AG, Basel
\end{abstract}

\section{Introduction}

First described in 1957 [1], acute promyelocytic leukemia (APL) is a subtype of acute myeloid leukemia (AML) characterized by the typical promyelocytic leukemia protein (PML)/retinoic acid receptor-a (RARA) rearrangement and an aggressive clinical presentation. In 1977, Rowley and colleagues [2] identified the characteristic $\mathrm{t}(15 ; 17)$ (q22;q12) balanced chromosomal translocation, while the pathophysiological effects of the deriving PML/ RARA fusion gene were described starting from the last decade of the 20th century [3] (Fig. 1).

Following description of the APL pathogenesis, the identification of targeted treatments, which first occurred "by chance," made APL a paradigm in the field of AML. Indeed, the specific PML/RARA rearrangement has a diagnostic and therapeutic role, not only as a drug target but also as a reliable biomarker for minimal residual disease monitoring, and for the definition of molecular relapse [4].

Dedicated to Prof. Francesco Lo-Coco, a great mentor and a pioneer in the studies on the pathogenesis of acute promyelocytic leukemia, the diagnosis, and the development of targeted treatments.

Carmelo Gurnari and Eleonora De Bellis contributed equally to this work.

\section{KARGER}

() 2020 S. Karger AG, Basel 


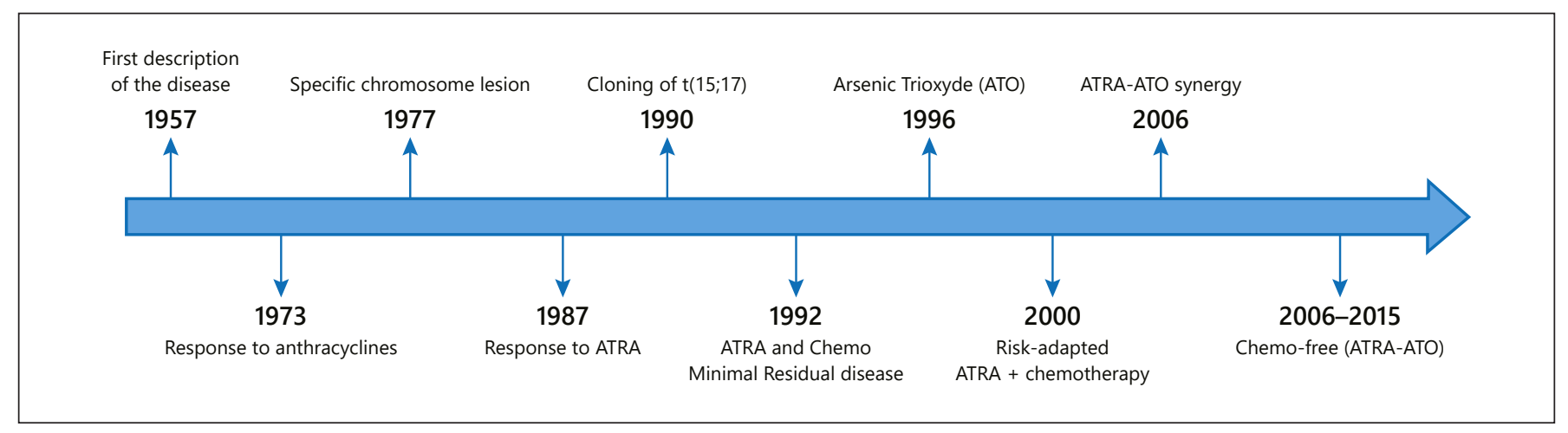

Fig. 1. Timeline of progresses in APL. ATO, arsenic trioxide; ATRA, all-trans-retinoic acid.

\section{APL Pathogenesis: The PML/RARA Fusion Gene}

The fusion of the N-terminus of the $P M L$, on chromosome 15 , to the C-terminus of the RARA, on chromosome 17, explains APL pathogenesis in $95-98 \%$ of cases, resulting in the production of the biological hallmark of the disease: the $P M L / R A R A$ rearrangement and the consequent oncogenic chimeric protein [5-7]. The prominent role of the rearrangement has been recently stressed by the updated 2016 WHO classification, which renamed this leukemia as "APL with PML-RARA" [8].

$\mathrm{PML}$ is a member of a protein family containing a zinc finger domain (RING domain), located within nuclear macromolecular structures, called PML-nuclear bodies (PML-NBs). PML acts as an organizer of PML-NBs and, in conditions of cellular stress, as a potent growth and tumor suppressor, exerting its proapoptotic properties through p53 activation. The interaction between PML and p53 results critical for senescence induction $[9,10]$.

RARA is a nuclear receptor that physiologically binds retinoic acid (RA), including natural or synthetic derivatives of vitamin A involved in cellular differentiation. RARA forms heterodimers with RXR and, in the absence of its ligands, recruits co-repressors and histone deacetylases to induce chromatin condensation and repression of transcription of target genes. In the presence of RA at physiological levels, the RARA-RXR heterodimer releases the repressor complex and recruits co-activators and histone acetyltransferases, resulting in hyperacetylation of histones at retinoic acid responsive element sites and accessibility of chromatin to transcription factors. In this setting, RARA becomes a potent activator of myeloid differentiation $[11,12]$. In the presence of the PML-RARA chimeric protein, the heterodimers bind more tightly the repressor complex and induce resistance to physiological levels of RA, constitutive repression of transcription, and the classical differentiation block seen in APL [13]. Pharmacological doses $(1 \mu \mathrm{M})$ of the RA derivative all-transretinoic acid (ATRA) are then able to degrade PMLRARA and release the repressor complex, reactivate transcription, and promote myeloid differentiation in vitro and in vivo (Fig. 2).

Although ATRA induces differentiation in all APL patients, it is not curative as a single agent, suggesting that impairment of differentiation is not the only mechanism sustaining the disease [14]. This is attributed to the double oncogenic pathway that underlies leukemogenesis in APL: the differentiation arrest and the aberrant self-renewal of APL cells. Indeed, PML-RARA interferes with the normal formation of PML-NBs, and this results in an impaired stress response and apoptosis block. In this setting, arsenic trioxide (ATO) has been shown to act on 2 fronts, inducing differentiation and apoptosis of APL cells. For these reasons, the combination of ATRA with chemotherapy and/or ATO is more effective than ATRA as monotherapy, in terms of long-term remission, both in de novo and relapsed cases $[11,12]$.

ATO acts through direct binding to PML/RARA via 2 cysteine residues (C212/213) in the B2 domain, inducing oxidation of these residues and formation of intermolecular disulfide bonds. This process leads to sumoylation of a lysine residue (K160) and to the reorganization of PMLPML/RARA from a diffuse/micro-speckled nuclear pool into PML-NBs. Within these structures, PML-PML/ RARA is polyubiquitinated by the SUMO-dependent E3 ubiquitin ligase ring finger protein 4 and successively degraded by the proteasome [15]. Besides inducing proteasome degradation, ATO also directly binds the wild-type PML proteins, promoting and amplifying the reformation of PML-NBs. Targeting of both PML-RARA and 


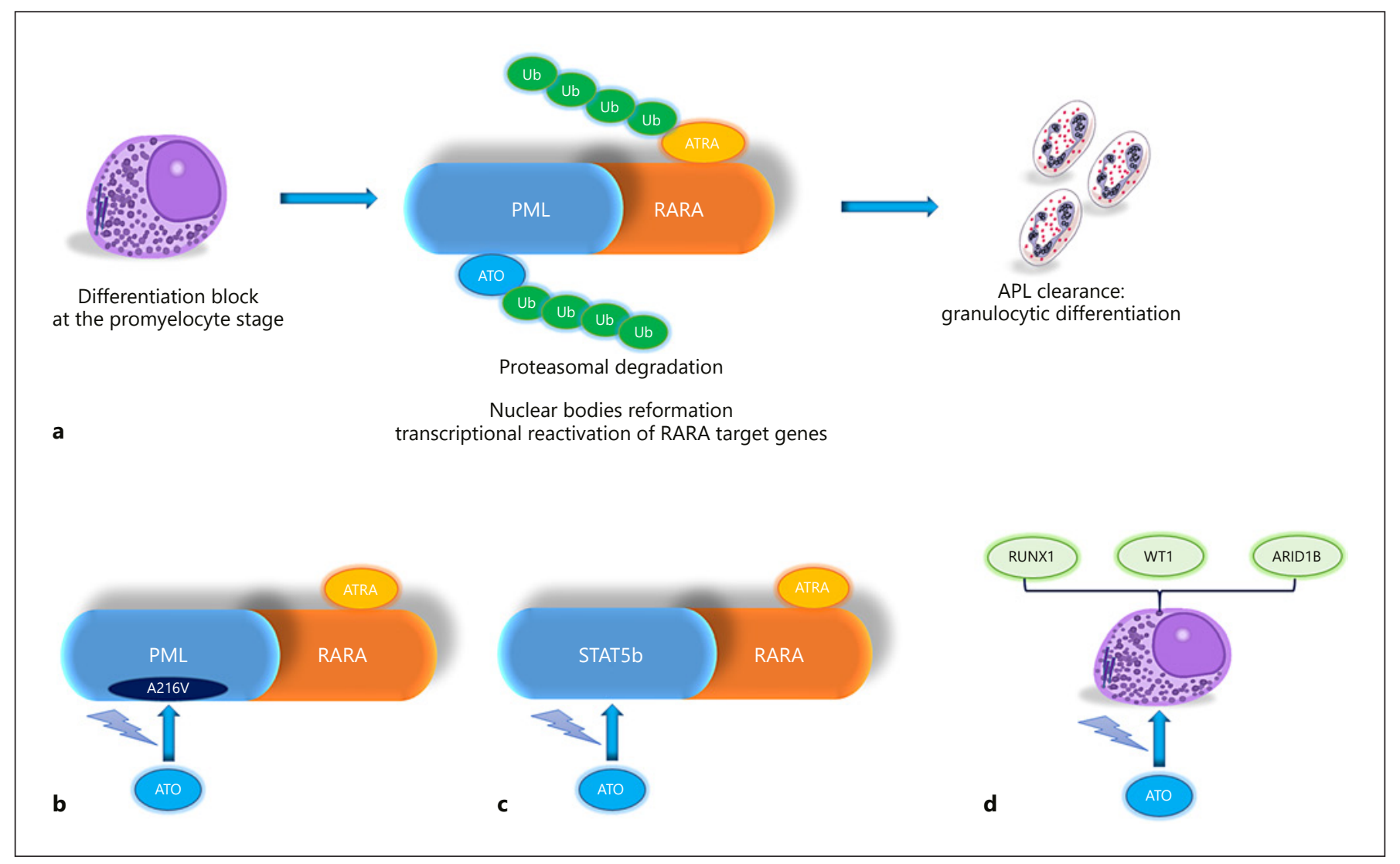

Fig. 2. Synergistic action of the combination ATRA/ATO and main mechanisms of resistance. a The PML/RARA fusion transcript blocks myeloid differentiation at the promyelocyte stage. The synergistic action of ATRA and ATO leads to proteasomal degradation of the oncoprotein, finally resulting in transcriptional reactivation of RARA target genes and granulocytic differentiation. b ATO resistance due to acquisition of mutations (A216V in the example) to alternative RARA rearrangement (with STAT5b in c), while the

wild-type PML by ATO results in a more effective proapoptotic activity and clinical superiority when added to ATRA [16]. Finally, ATO is able to degrade PML-RARA through an indirect mechanism, recruiting caspases and inducing production of reactive oxygen species, which are toxic for APL cells.

\section{History of Arsenic as a Drug}

Interestingly, the clinical application of ATO in APL preceded the complete understanding of its molecular mechanisms of action. Actually, several arsenic-containing regimens have been used for a long time, both in Western medicine and in Chinese traditional medicine presence of additional somatic mutations in APL cells is a more prominent feature in patients treated with the ATRA/chemotherapy combination (d). PML, promyelocytic leukemia protein; RARA, retinoic acid receptor-a; APL, acute promyelocytic leukemia; ATRA, all-trans-retinoic acid; ATO, arsenic trioxide; Ub, ubiquitin; STAT5b, signal transducer and activator of transcription 5b; ARID1B, AT-rich interaction domain 1B; RUNX1, runt-related transcription factor 1; WT1, Wilms' tumor gene 1.

[17]. At the same time, arsenic has a long history as poison to humans, both for intentional and environmental exposure. In the environment, arsenic is found in inorganic and organic forms and in different valence or oxidation states, being the trivalent oxidation state (which ATO belongs to) characterized by the strongest toxicity. Considered the "king of poisons," arsenic gained notoriety as an intentional homicidal or suicidal poison during the Middle Ages and Renaissance because of its odorless and tasteless properties and its frequent involvement in high-personality murders. Medical recipes containing arsenic are even more ancient, and it is thought that Hippocrates already used an arsenic paste to treat ulcers and abscesses [18]. In 1786, Thomas Fowler introduced a potassium bicarbonate-based solution of ATO that was em- 
pirically used in the treatment of various diseases, including malaria, syphilis, asthma, chorea, eczema, and psoriasis. After leukemia was discovered in 1845, because of its capability to lower white blood cell (WBC) counts, "Fowler's solution" was used to treat leukemia first in 1865 and again in 1931 [19]. In 1910, Paul Ehrlich discovered a new arsenic-based drug called "Salvarsan," which was used to treat syphilis until the advent of penicillin [20]. After a decline in the use of arsenic due to its excessive toxicity, in the 1960s, the Chinese traditional medicine started to use combinations including arsenic sulfate to treat leukemia. Since the preparation and processing of these medical recipes did not involve the high temperature required for the conversion of arsenic sulfate into ATO, the relation of arsenic sulfate with ATO in such composites is unclear [17].

A new Chinese arsenic-based drug to treat cancers appeared in 1971, called "713" or "Ailin," consisting of a combination of ATO, mercury, and toad venom. In the subsequent years, Zhang and colleagues focused their research on the treatment of leukemia and, after having analyzed the components of "713," they observed that the therapeutic effect was solely ascribable to arsenic. Since then, the recipe was modified, leaving only ATO ("Ailin I") and a trace amount of mercury, and in 1979, it was successfully used to treat patients with acute leukemia, with granulocytic leukemia (corresponding to the Western definition of APL) being the most sensitive to the treatment. In 1991, Sun and colleagues continued the work of Zhang and reported that "Ailin I" had been used from 1974 to 1985 to treat 32 patients with APL, with achievement of remission in 19 cases and long-term survival in 16 cases [17].

In the same decades, the effects of ATRA on myeloid differentiation of APL cells were reported first in vitro and then in vivo, leading to the recognition of the role of ATRA in APL treatment in 1991 [21-23]. As reported by Rao et al. [17], first studies on the in vitro effects of ATO on leukemic cells were published in 1992, and in 1996, Chen et al. [24] reported apoptosis induction as one of the possible mechanisms of ATO on the APL clone. After 1 year, the group of Shanghai published the dose-dependent effects of ATO on leukemic cells in vitro: at higher concentrations (0.5-2 mM), ATO preferentially induces apoptosis, mainly through activation of the mitochondria-mediated intrinsic apoptotic pathway, while at lower concentrations (0.1-0.5 mM), ATO promotes the partial differentiation of APL cells, both in ATRA-sensitive or ATRA-resistant APL cells [25]. This study laid the foundations for understanding the complex and dualistic mechanisms of action of ATO on APL cells. Meanwhile, two research groups demonstrated the synergic superiority and curative potential of ATRA-ATO combination therapy using transgenic mouse models of APL [26, 27]. In 2000, ATO was also approved by the FDA for the treatment of relapsed APL.

\section{Clinical Use of Arsenic in APL: The Long Journey to a Chemotherapy-Free Approach}

Until recently, ATRA combined with anthracyclines has been the standard of care for newly diagnosed patients with APL [28]. With this strategy, about 70-75\% long-term overall survival rates have been achieved. However, myelosuppression and subsequent infections, and early and late toxicities (including secondary leukemias) were major concerns. In this scenario, early studies conducted in China and the USA demonstrated that single-agent ATO could induce molecular remission in approximately $80 \%$ of APL patients, relapsed after ATRA chemotherapy regimens [29-31] (Table 1). These results in the relapsed setting paved the way for the use of ATO in newly diagnosed patients with APL. Shen et al. [32] proved for the first time that the ATRA/ATO combination exerted a synergic action in APL, showing that none of 20 patients treated with the ATRA/ATO combination relapsed, while 7 of 37 cases (19\%) in the monotherapy group relapsed after a median follow-up of 18 months. Riding the wave of a more effective and chemo-free alternative to ATRA plus anthracyclines, Estey et al. [33] demonstrated the feasibility of the ATRA/ATO approach in newly diagnosed patients with APL of all-risk groups [34]. Unlike Shen et al. [32], the study included the use of gemtuzumab ozogamicin in case of high-risk groups (WBC count exceeding 10,000 $\times 10^{9} / \mathrm{L}$, PML-RARA PCR positive after the $3 \mathrm{rd}$ month from complete remission or any toxicity related to ATRA/ATO treatment). A total of 44 patients received ATRA ( $45 \mathrm{mg} / \mathrm{m}^{2}$ daily) and ATO $(0.15 \mathrm{mg} / \mathrm{kg}$ daily, beginning on day 10 of ATRA) as induction therapy, and for 28 weeks after complete remission (CR), with dose adjustments according to treatment toxicity. Overall, the CR rate was $89 \%$ (96\% in standardrisk and 79\% in high-risk APL), proposing this combination as an alternative treatment to chemotherapy in standard-risk APL. Following this idea, Iland et al. [35] (APLM4 study) included ATO during both induction and consolidation in patients with newly diagnosed APL, achieving excellent survival outcomes despite a substantial reduction in the dose of chemotherapy and confirming previously reported results of improved event-free 
Table 1. Results of arsenic treatment in APL according to the major clinical trials and case series

\begin{tabular}{|c|c|c|c|c|c|c|c|}
\hline $\begin{array}{l}\text { APL } \\
\text { type }\end{array}$ & Patients, $n$ & $\begin{array}{l}\text { Arsenic dose, } \\
\mathrm{mg} / \mathrm{kg}\end{array}$ & $\begin{array}{l}\text { Prevalence of } \\
\text { differentiation } \\
\text { syndrome, \% }\end{array}$ & $\begin{array}{l}\text { Median } \\
\text { follow-up } \\
\text { time, } \\
\text { months }\end{array}$ & $\begin{array}{l}\text { EFS or } \\
\text { DFS, \% }\end{array}$ & OS, \% & Ref. \\
\hline \multirow[t]{2}{*}{$\begin{array}{l}\text { Standard } \\
\text { risk }\end{array}$} & 77 pts: first-line ATRA/ATO & 0.15 & 19 & 66.4 & $\begin{array}{l}\text { EFS: } 96.6 ; \\
\text { DFS: } 96.6\end{array}$ & 99 & $\begin{array}{l}{[38,} \\
40]\end{array}$ \\
\hline & 37 (ATRA/ATO) versus 72 (ATRA/RIF) pts: first-line & $\begin{array}{l}\text { ATO } 0.16 \\
\text { versus RIF } 60\end{array}$ & $\begin{array}{l}28 \text { (ATO); } \\
26 \text { (RIF) }\end{array}$ & 32 & $\begin{array}{l}\text { EFS: } 94 \text { (ATO); } \\
97 \text { (RIF) }\end{array}$ & $\begin{array}{l}94 \text { (ATO); } \\
100 \text { (RIF) }\end{array}$ & [59] \\
\hline \multirow{8}{*}{$\begin{array}{l}\text { Standard } \\
\text { and high } \\
\text { risk }\end{array}$} & 40 relapsed pts: ATO alone & NA & 25 & 18 & 56 & 66 & [31] \\
\hline & 20 pts: first-line ATRA/ATO & 0.16 & 0 & 18 & DFS: 95 & 100 & [32] \\
\hline & $\begin{array}{l}44 \text { pts: first-line ATRA/ATO ( } 19 \mathrm{HR} \text { pts + GO or IDA } \\
\text { during induction) }\end{array}$ & 0.15 & 20 & 16 & RFS: 92 & 86 & [33] \\
\hline & 116 pts: first-line ATRA/ATO (28 HR pts + GO) & $\begin{array}{l}0.3 \text { and then } \\
0.25\end{array}$ & 25.8 & 30.5 & EFS: 91 & 93 & [43] \\
\hline & 187 pts: first-line ATRA/ATO \pm GO (54 HR pts) & 0.15 & 11 & 47.6 & $\begin{array}{l}\text { EFS: } 85 \text {; } \\
\text { DFS: } 96\end{array}$ & 88 & [41] \\
\hline & $\begin{array}{l}278 \text { pts: first-line ATRA/AraC/IDA + consolidation with } \\
\text { ATO ( } 98 \text { HR pts) }\end{array}$ & 0.15 & NA & 52 & $\begin{array}{l}\text { EFS: } 95.7(\mathrm{SR}) \\
92.1 \text { (HR) }\end{array}$ & $\begin{array}{l}95.7(\mathrm{SR}) \\
93(\mathrm{HR})\end{array}$ & {$[44]$} \\
\hline & $\begin{array}{l}101 \text { pediatric pts: first-line ATRA/IDA + consolidation } \\
\text { with ATO }\end{array}$ & 0.15 & 20 & 44.7 & $\begin{array}{l}\text { EFS: } 95 \text { (SR); } \\
83 \text { (HR) }\end{array}$ & $\begin{array}{l}98 \text { (SR); } \\
86 \text { (HR) }\end{array}$ & [48] \\
\hline & 18 pediatric pts: first-line ATRA/ATO & 0.15 & 5.5 & 24 & DFS: 100 & 100 & {$[50]$} \\
\hline
\end{tabular}

SR, standard (low-to-intermediate)-risk APL according to the Sanz criteria; HR, high-risk APL according to the Sanz criteria; ATO, arsenic trioxide; RIF, Realgar-Indigo naturalis formula; ATRA, all-trans-retinoic acid; IDA, idarubicin; AraC, cytarabine; DAUNO, daunorubicin; GO, gemtuzumab ozogamicin; EFS, event-free survival; DFS, disease-free survival; OS, overall survival; NA, not available; pts, patients.

survival (EFS) when using ATO in the consolidation phase [36]. The APLM4 study [35] also confirmed the observations by Mathews et al. [37], who noticed that ATO may abrogate the poor outcome of patients harboring FLT3 mutations. Since that moment, all the studies variably included ATO in both induction and consolidation phases, in combination with other cytotoxic agents (idarubicin, daunorubicin, and gemtuzumab). The role of maintenance in the new ATO era remained a matter of controversial debate.

Many of the open issues were then solved by the practice-changing Italian-German study APL0406, which confirmed the combination of ATRA and ATO as the reference treatment for standard-risk APL [38]. This multicenter phase 3 trial compared ATRA plus chemotherapy with ATRA plus ATO in newly diagnosed patients with standard-risk APL. The first results showed that the chemo-free combination provided an advantage in terms of
EFS, probably as a consequence of the reduced severity of myelosuppression, together with the increased antileukemic efficacy. Moreover, the toxicity profile of ATRA/ATO appeared to be mild and manageable if compared to the previously experienced toxicities of ATRA plus chemotherapy. Indeed, myelosuppression was milder in the ATRA/ATO group, and consequently fewer febrile neutropenia episodes were recorded (30 vs. 75 in the ATRA/ ATO and ATRA/chemotherapy arms, respectively, $p<$ 0.001). The major nonhematological toxicities in the ATRA/ATO group were transient liver enzyme alterations (44 vs. $3 \%$ ) and QTc prolongation (11 vs. $0.7 \%$ ), while gastrointestinal and cardiac function abnormalities were more frequent in the ATRA/chemotherapy group. A typical aspect of the ATRA/ATO group was the development of leukocytosis during induction ( $43 \%$ of cases), successfully managed with hydroxyurea (the only cytotoxic agent allowed as per protocol recommendation). Differ- 
ent from the previous studies, where ATO was started at day 9 or 10 of induction to reduce the risk of hyperleukocytosis and subsequent differentiation syndrome (DS) $[33,35]$, in the APL0406 study, ATO was administered at the beginning of the induction phase. Moderate to severe DS [39] was registered in 19\% of patients in the ATRA/ ATO group and $16 \%$ in the ATRA/chemotherapy group. Dexamethasone treatment $(10 \mathrm{mg}$ every $12 \mathrm{~h}$ for at least 3 days) was used at the first suspicion of DS. The excellent outcomes of the chemo-free regimen were also emphasized by the recent update [40] of this pivotal study, showing an increased advantage over time of the ATRA/ATO combination in terms of EFS ( $96.6 \%$ at 72 months vs. $77.4 \%$ in the ATRA/chemotherapy group), and a significantly reduced cumulative incidence of relapse (1.7 vs. $15.5 \%$, respectively). The long-lasting advantage of the chemo-free regimen, concentrating the treatment in about 7 months, doubted the role of maintenance, especially pointing out the $2 \%$ increased prevalence of secondary leukemias in the ATRA/chemotherapy arm. The low incidence of relapses, which occurred exclusively during the first 24 months in the ATRA/ATO arm, also questioned the necessity of prolonged molecular monitoring in patients with standard-risk APL treated with ATO frontline. The durability of the results of the ATRA/ATO combination was confirmed by Abaza et al. [41], who also demonstrated that relapses concentrate in the first year after CR. Indeed, we [42] showed that ATRA/ATO exerts superior antileukemic activity compared to ATRA/chemotherapy, by significantly reducing the PML-RARA load during treatment and monitoring, irrespective of FLT3 mutational status. Moreover, we also confirmed the lack of prognostic significance of minimal residual disease levels at the post-induction checkpoint, emphasizing instead the relevance of the post 3rd consolidation time point.

If these results showed that ATRA/ATO combination is the standard of care for low-to-intermediate risk patients, there is still room to improve the treatment schedule of high-risk patients (about $25 \%$ of newly diagnosed APL). The randomized AML17 study [43], conducted in the UK, showed that ATRA/ATO is feasible also in this setting. The ATRA treatment scheme was similar to APL0406, with this drug given orally at the same dose for the first 60 days or until remission, and then for 15 days on an on-and-off basis (for days 1-14 and 29-42 during courses 2-4 and for days 1-14 of course 5). On the other hand, ATO was given intravenously at the alternative dose of $0.3 \mathrm{mg} / \mathrm{kg}$ on days $1-5$ of each course, and then at $0.25 \mathrm{mg} / \mathrm{kg}$ twice weekly (during the rest of induction and weeks $2-4$ of courses 2-5). Actually, the ATRA/ATO combination prolonged the EFS also in high-risk APL, when compared to ATRA/chemotherapy ( 87 vs. $64 \%$ at 4 years, respectively, $p=0.07$ ). Furthermore, the new schedule of administration of ATO (with 63/168 treatment days vs. 140/180 days, and a twice-weekly administration) led to a lower incidence of liver toxicity (around $29 \%$ of grade 3-4 toxicity, compared to $40 \%$ in the ItalianGerman trial during induction). A further difference from the APL0406 trial was the elimination of maintenance therapy. Similar to previous studies [33, 41], highrisk patients received a dose of gemtuzumab ozogamicin $\left(6 \mathrm{mg} / \mathrm{m}^{2}\right)$ within the first 4 days of induction, to lower WBC counts and prevent the risk of hyperleukocytosis and subsequent DS. Using these precautions, the incidence of DS was similar in high- and standard-risk patients. The results of the AML17 study have been further confirmed by the APL2006 trial, conducted by the French Belgian Swiss APL group in 795 patients of all-risk groups to test the role of ATO in the consolidation phase [44]. After a classical ATRA/chemotherapy induction, 214 high-risk patients were randomized to receive either chemotherapy or chemotherapy plus ATO. The 5-year EFS was 85.5 and $92.1 \%(p=0.38)$ in the chemotherapy alone and in the chemotherapy plus ATO group, respectively. Of note, during conduction of the study, an amendment led to the exclusion of cytarabine in the ATO group due to the high rate of myelosuppression. This study showed that ATO can replace chemotherapy in high-risk patients, without increasing the risk of relapse and with a milder toxicity profile in terms of myelosuppression and infectious episodes. The randomized, multicenter, pan-European APOLLO trial (NCT02688140) and a Chinese study [45] are ongoing to specifically assess the role of ATO/ ATRA frontline in high-risk APL.

The introduction of ATO in the panoply of APL management also improved patient-reported outcomes and quality of life. Actually, in the Italian-German study [46], lower fatigue severity was reported at the end of induction in patients receiving ATRA/ATO versus ATRA/chemotherapy (mean score difference, -9.3 ; $95 \% \mathrm{CI},-17.8$ to $-0.7 ; p=0.034)$. Results at long term are being exploited and will be also compared to the recent Italian study [47] conducted in APL survivors treated with standard chemotherapy, showing that, after a median follow-up of 14.3 years from diagnosis, the symptom "fatigue" was reported as moderate to severe by $29 \%$ of patients, with $84.4 \%$ of patients complaining at least one comorbidity. The new formulation of arsenic (see below) may further improve treatment results and allow a chemo-free, totally oral anti-APL regimen. 


\section{Special Situations in APL}

Since the introduction of ATRA/ATO as standard of care in APL, some hurdles remain to face. As previously discussed, arsenic has been mostly used in randomized trials including patients aged between 18 and 70 years, leaving elderly patients and children as patient categories reported as sporadic cases or retrospective series. Indeed, the recent experiences with ATRA/ATO combinatory trials in adults prompted pediatricians to translate this highly effective frontline treatment in newly diagnosed children with APL. The COG-AAML0631 protocol, including 101 children receiving induction therapy with ATRA/ idarubicin and ATRA/ATO as consolidation for both high- and standard-risk APL, showed that ATO may reduce the cumulative anthracycline dose, without affecting survival outcomes [48]. This led to the use of the chemo-free combination frontline in children, with excellent results reported by different groups, without known longterm sequelae $[49,50]$. The main difference with adults is the lower ATRA dose $\left(25 \mathrm{mg} / \mathrm{m}^{2}\right)$, due to the recognized higher risk of pseudotumor cerebri in the younger population [51]. A new open-label protocol (ICC APL Study 02, Eudract: 2017-002383-40) investigating ATRA/ATO frontline is now ongoing to validate these results in the standard-risk setting.

Similarly, elderly patients have been mostly investigated after the introduction of ATO in a scattered and retrospective fashion $[43,52]$. Older patients with APL (aged $>70$ years) treated with chemotherapy are more prone to develop toxicities and experience up to $60 \%$ early death rates [53], making the milder toxicity profile of ATO combinations attractive to treat these fragile patients. A large retrospective study analyzed data from 475 patients (median age 73.4 years, range $70-89$ years) derived from different multinational registries. Patients received different supportive therapies, and only 26 patients $(6 \%)$ were treated with the ATRA/ATO regimen, while the rest received ATO in different combinations with ATRA and chemotherapy. The early death rate was lower for the ATRA/ATO combinations (8 vs. 18\%), while the CR rate was $82 \%$. The estimated 5 -year overall survival within the wide 1990-2018 temporal range was $66 \%$. A recent abstract [54] presented at the 2019 ASH meeting, reporting real-life data on 45 patients aged $>70$ years, showed that at least half of them were not considered eligible for a standard approach, highlighting the unmet clinical need of protocols specific for this patient subset, where also different ATO scheduling and dosing could be tested.

\section{Oral ATO Formulations}

In the long journey toward a more effective, manageable, and toxicity-free regimen, the availability of an oral arsenic compound has been eagerly pursued in APL. Again, Chinese researchers have been pioneers in testing oral arsenic compounds [55]. Nowadays, the only commercially available agent in China is RealgarIndigo naturalis formula (RIF), approved by the Chinese Food and Drug Administration in 2009 for the treatment of APL [56]. First developed in 1980, RIF demonstrated in vitro and in vivo activity, due to the upregulation of aquaglyceroporin 9 that allows its intracellular uptake [57]. So far, more than 5,000 patients have been treated with RIF-based regimens with excellent results and very favorable toxicity profiles, especially when considering the potential of a completely chemo-free regimen [55]. An important milestone has been the demonstration of non-inferiority of ATRA/ ATO versus ATRA/RIF treatment in terms of 2-year EFS in newly diagnosed patients with APL (94 vs. 97\%, respectively) [58]. These results were confirmed by the use of RIF/ATRA in standard-risk and later in high-risk patients $[59,60]$. Regarding toxicities, the favorable pharmacokinetic profile (reaching a more wide and prolonged concentration curve) renders the oral formulation attractive in terms of cardiac and liver toxicity, main drawbacks of the i.v. ATO formulation. If up to $16 \%$ of patients with APL experience a QTc prolongation of any grade after ATO administration [38], no cardiac toxicity has been reported using $\operatorname{RIF}[59,60]$. On the other hand, liver toxicity still occurs with ATRA/ RIF, at a milder degree than with ATRA/ATO [58]. A further advantage of the ATRA/RIF combination is the applicability of this entirely chemotherapy-free, outpatient-based post-remission strategy, attractive in terms of quality of life and health system-related costs, in particular for children or elderly patients [61].

\section{ATO: Mechanisms of Resistance}

The most frequent mechanisms of ATRA/ATO resistance are due to the presence of alternative genetic rearrangements in cases of AML with an APL-like morphology. Indeed, a minor proportion of APL-like AML does not present the typical $P M L-R A R A$ fusion gene: in these cases, variant cytogenetic translocations have been identified, involving the RARA gene on chromosome 17 and alternative partner genes, resulting in $X-R A R A$ re- 
arrangements. The most common and best known "APL variant" presents the PLZF-RARA fusion gene, as a result of the $\mathrm{t}(11 ; 17)(\mathrm{q} 23 ; \mathrm{q} 21)$ chromosomal translocation, first described by Chen and colleagues [62]. The second most common "APL variant" is characterized by the NPM1-RARA rearrangement, caused by the $\mathrm{t}(5 ; 17)$ (q35;q21) translocation. Other partner genes, alternative to $P M L$, have been observed in few case reports; among them, $S T A T 5 b$ is of interest, since its presence, similar to PLZF, is synonymous of resistance to ATRAATO therapy and worse prognosis (Fig. 2) [63]. The lack of ATO-binding sites in X-RARA oncogenic proteins is thought to be responsible for ATO resistance [64].

An additional molecular mechanism of resistance to ATO is the presence of mutations within the B2 domain of the PML gene, where the ATO-binding region is located. Lack of response to ATO has been documented in vitro and in vivo in APL cells harboring missense mutations in this region $[65,66]$. The most common mutation results from the $\mathrm{A} 216 \mathrm{~V}$ amino acidic substitution in the B2 domain, which we found not only in the PML-RARA fusion gene but also in the unrearranged $P M L$ allele, although in different clones. In these cases, ATO binding is prevented, and this impairs the disruption of the oncogenic fusion protein and the reformation of NBs, which are the 2 mechanisms of action of this drug [67] (Fig. 2). These types of mutations, as well as those observed in the RARA gene, which impair response to ATRA, mostly emerge under the selective pressure of ATO treatment [68-70].

We also found that patients relapsing after ATRAATO treatment are characterized by a higher number of mutations in other genes, including ASXL1, DNMT3A, JAK2, SRSF2, TET2, and TP53 [70]. Similarly, Madan et al. [71] found FLT3-ITD, WT1, RUNX1, and ARID1B mutations as recurrent in relapsing APL cases. However, the appearance of additional somatic mutations in resistant/relapsed APL is a more prominent feature in patients previously treated with the ATRA-chemotherapy combination (Fig. 2).

Other possible mechanisms of resistance to ATO are under study and are not yet clear. Involvement of the microenvironment has been hypothesized, together with alterations in the redox system and abnormalities in autophagy pathways, considering that some autophagy regulatory proteins have been shown to play a prosurvival role in APL cells during ATRA-ATO treatment [64].

\section{Conclusions and Future Perspectives}

Arsenic has shown to be a very effective drug in APL. This compound allowed the management of a highly fatal hematological cancer with a totally chemo-free approach, with impressive survival rates. However, many issues still require international efforts. For example, the role of oral arsenic, possibly leading to management of APL on an outpatient basis at least in the post-induction phase, will have to be confirmed by the ongoing and clinical trials. Together with ATRA, arsenic is one of the first examples of precision medicine and, in the near future, the availability of big data will help further dig into the still open contentions in APL, including the long-term effects of arsenic in the increasing population of APL survivors.

\section{Disclosure Statement}

The authors declare no conflicts of interest.

\section{Funding Sources}

This work was supported by the AIRC $5 \times 1,000$ call "Metastatic disease: the key unmet need in oncology" to MYNERVA project, \#21267 (MYeloid NEoplasms Research Venture AIRC; a detailed description of the MYNERVA project is available at http:// www.progettoagimm.it), by GR-2018-12365529-Santa Lucia to T.O.; PRIN Grant No. 2017WXR7ZT to M.T.V.; and the Innovative Medicines Initiative (IMI) 2 project "HARMONY," No. 116026 , to M.T.V.

\section{Author Contributions}

C.G. and E.D wrote the manuscript. M.D., T.O., and S.V. edited the manuscript. M.T.V. conceived the idea and wrote the manuscript. All coauthors revised the manuscript.

References

1 Hillestad LK. Acute promyelocytic leukemia. Acta Med Scand. 1957 Nov 29;159(3):189-94.

2 Rowley JD, Golomb HM, Dougherty C. 15/17 translocation, a consistent chromosomal change in acute promyelocytic leukaemia. Lancet. 1977 Mar 5;1(8010):549-50.

3 Pandolfi PP, Alcalay M, Fagioli M, Zangrilli D, Mencarelli A, Diverio D, et al. Genomic variability and alternative splicing generate multiple PML/RAR alpha transcripts that encode aberrant PML proteins and PML/ RAR alpha isoforms in acute promyelocytic leukaemia. Embo J. 1992 Apr;11(4):1397407. 
4 Sanz MA, Grimwade D, Tallman MS, Lowenberg B, Fenaux P, Estey EH, et al. Management of acute promyelocytic leukemia: recommendations from an expert panel on behalf of the European LeukemiaNet. Blood. 2019 Apr 11;113(9):1875-91.

5 de Thé H, Chomienne C, Lanotte M, Degos L, Dejean A. The $\mathrm{t}(15 ; 17)$ translocation of acute promyelocytic leukaemia fuses the retinoic acid receptor alpha gene to a novel transcribed locus. Nature. 1990 Oct 11; 347(6293):558-61.

6 Borrow J, Goddard AD, Gibbons B, Katz F, Swirsky D, Fioretos T, et al. Diagnosis of acute promyelocytic leukaemia by RT-PCR: detection of PML-RARA and RARA-PML fusion transcripts. Br J Haematol. 1992 Nov;82(3): $529-40$.

7 Borrow J, Solomon E. Molecular analysis of the $t(15 ; 17)$ translocation in acute promyelocytic leukaemia. Baillieres Clin Haematol. 1992 Oct;5(4):833-56.

8 Arber DA, Orazi A, Hasserjian R, Thiele J, Borowitz MJ, Le Beau MM, et al. The 2016 revision to the World Health Organization classification of myeloid neoplasms and acute leukemia. Blood. 2016 May 19;127(20):2391405.

9 Guo A, Salomoni P, Luo J, Shih A, Zhong S, $\mathrm{Gu} W$, et al. The function of PML in p53-dependent apoptosis. Nat Cell Biol. 2000 Oct; 2(10):730-6.

10 de Thé $\mathrm{H}$. Differentiation therapy revisited. Nat Rev Cancer. 2018 Feb;18(2):117-27.

11 Dos Santos GA, Kats L, Pandolfi PP. Synergy against PML-RARa: targeting transcription, proteolysis, differentiation, and self-renewal in acute promyelocytic leukemia. J Exp Med. 2013 Dec 16;210(13):2793-802.

12 Lo-Coco F, Hasan SK. Understanding the molecular pathogenesis of acute promyelocytic leukemia. Best Pract Res Clin Haematol. 2014 Mar;27(1):3-9.

13 Kwok C, Zeisig BB, Dong S, So CW. Forced homo-oligomerization of RARalpha leads to transformation of primary hematopoietic cells. Cancer Cell. 2006 Feb;9(2):95-108.

14 Nasr R, Guillemin MC, Ferhi O, Soilihi H, Peres L, Berthier C, et al. Eradication of acute promyelocytic leukemia-initiating cells through PML-RARA degradation. Nat Med. 2008 Dec;14(12):1333-42.

15 Lallemand-Breitenbach V, Jeanne M, Benhenda S, Nasr R, Lei M, Peres L, et al. Arsenic degrades PML or PML-RARalpha through a SUMO-triggered RNF4/ubiquitin-mediated pathway. Nat Cell Biol. 2008 May;10(5):547-55.

16 Ablain J, Rice K, Soilihi H, de Reynies A, Minucci S, de Thé $\mathrm{H}$. Activation of a promyelocytic leukemia-tumor protein 53 axis underlies acute promyelocytic leukemia cure. Nat Med. 2014 Feb;20(2):167-74

17 Rao Y, Li R, Zhang D. A drug from poison: how the therapeutic effect of arsenic trioxide on acute promyelocytic leukemia was discovered. Sci China Life Sci. 2013 Jun;56(6):495502.
18 Hughes MF, Beck BD, Chen Y, Lewis AS, Thomas DJ. Arsenic exposure and toxicology: a historical perspective. Toxicol Sci. 2011 Oct; 123(2):305-32.

19 Jolliffe DM. A history of the use of arsenicals in man. J R Soc Med. 1993 May;86(5):287-9.

20 Antman $\mathrm{KH}$. Introduction: the history of arsenic trioxide in cancer therapy. Oncologist. 2001;6(Suppl 2):1-2.

21 Koeffler HP. Induction of differentiation of human acute myelogenous leukemia cells: therapeutic implications. Blood. 1983 Oct; 62(4):709-21.

22 Warrell RP Jr, Frankel SR, Miller WH Jr, Scheinberg DA, Itri LM, Hittelman WN, et al. Differentiation therapy of acute promyelocytic leukemia with tretinoin (all-trans-retinoic acid). N Engl J Med. 1991 May 16;324(20): 1385-93.

23 Huang M, Ye Y, Chen S, Chai J, Lu J, Zhoa L, et al. Use of all-trans retinoic acid in the treatment of acute promyelocytic leukemia. Blood. 1988;72(2):567-72. Blood. 2016 Dec 29; 128(26):3017. [Check Journal Title]

24 Chen GQ, Zhu J, Shi XG, Ni JH, Zhong HJ, Si $\mathrm{GY}$, et al. In vitro studies on cellular and molecular mechanisms of arsenic trioxide (As2O3) in the treatment of acute promyelocytic leukemia: As2O3 induces NB4 cell apoptosis with downregulation of $\mathrm{Bcl}-2$ expression and modulation of PML-RAR alpha/PML proteins. Blood. 1996 Aug 1;88(3):1052-61.

25 Chen GQ, Shi XG, Tang W, Xiong SM, Zhu J, Cai X, et al. Use of arsenic trioxide (As2O3) in the treatment of acute promyelocytic leukemia (APL): I. As2O3 exerts dose-dependent dual effects on APL cells. Blood. 1997 May 1; 89(9):3345-53.

26 Lallemand-Breitenbach V, Guillemin MC, Janin A, Daniel MT, Degos L, Kogan SC, et al. Retinoic acid and arsenic synergize to eradicate leukemic cells in a mouse model of acute promyelocytic leukemia. J Exp Med. 1999 Apr 5;189(7):1043-52.

27 Rego EM, He LZ, Warrell RP Jr, Wang ZG, Pandolfi PP. Retinoic acid (RA) and As2O3 treatment in transgenic models of acute promyelocytic leukemia (APL) unravel the distinct nature of the leukemogenic process induced by the PML-RARalpha and PLZFRARalpha oncoproteins. Proc Natl Acad Sci U S A. 2000 Aug 29;97(18):10173-8.

28 Sanz MA, Grimwade D, Tallman MS, Lowenberg B, Fenaux P, Estey EH, et al. Management of acute promyelocytic leukemia: recommendations from an expert panel on behalf of the European LeukemiaNet. Blood. 2009 Feb 26;113(9):1875-91.

29 Cortes JE, Kantarjian H, O’Brien S, Robertson LE, Koller C, Hirsh-Ginsberg C, et al. Alltrans retinoic acid followed by chemotherapy for salvage of refractory or relapsed acute promyelocytic leukemia. Cancer. 1994 Jun 15; 73(12):2946-52.

30 Shen ZX, Chen GQ, Ni JH, Li XS, Xiong SM, Qiu QY, et al. Use of arsenic trioxide (As2O3) in the treatment of acute promyelocytic leu- kemia (APL): II. Clinical efficacy and pharmacokinetics in relapsed patients. Blood. 1997 May 1;89(9):3354-60.

31 Soignet SL, Frankel SR, Douer D, Tallman MS, Kantarjian H, Calleja E, et al. United States multicenter study of arsenic trioxide in relapsed acute promyelocytic leukemia. J Clin Oncol. 2001 Sep 15;19(18):3852-60.

32 Shen ZX, Shi ZZ, Fang J, Gu BW, Li JM, Zhu $\mathrm{YM}$, et al. All-trans retinoic acid/As2O3 combination yields a high quality remission and survival in newly diagnosed acute promyelocytic leukemia. Proc Natl Acad Sci U S A. 2004 Apr 13;101(15):5328-35.

33 Estey E, Garcia-Manero G, Ferrajoli A, Faderl S, Verstovsek S, Jones D, et al. Use of all-trans retinoic acid plus arsenic trioxide as an alternative to chemotherapy in untreated acute promyelocytic leukemia. Blood. 2006 May 1; 107(9):3469-73.

34 Sanz MA, Lo Coco F, Martín G, Avvisati G, Rayón C, Barbui T, et al. Definition of relapse risk and role of nonanthracycline drugs for consolidation in patients with acute promyelocytic leukemia: a joint study of the PETHEMA and GIMEMA cooperative groups. Blood. 2000 Aug 15;96(4):1247-53.

35 Iland HJ, Bradstock K, Supple SG, Catalano A, Collins M, Hertzberg M, et al. All-transretinoic acid, idarubicin, and IV arsenic trioxide as initial therapy in acute promyelocytic leukemia (APML4). Blood. 2012 Aug 23; 120(8):1570-752; quiz 752.

36 Powell BL, Moser B, Stock W, Gallagher RE Willman CL, Stone RM, et al. Arsenic trioxide improves event-free and overall survival for adults with acute promyelocytic leukemia: North American Leukemia Intergroup Study C9710. Blood. 2010 Nov 11;116(19):3751-7.

37 Mathews V, Thomas M, Srivastava VM, George B, Srivastava A, Chandy M. Impact of FLT3 mutations and secondary cytogenetic changes on the outcome of patients with newly diagnosed acute promyelocytic leukemia treated with a single agent arsenic trioxide regimen. Haematologica. 2007 Jul;92(7): 994-5.

38 Lo-Coco F, Avvisati G, Vignetti M, Thiede C, Orlando SM, Iacobelli S, et al. Retinoic acid and arsenic trioxide for acute promyelocytic leukemia. N Engl J Med. 2013 Jul 11; 369(2):111-21.

39 Montesinos P, Bergua JM, Vellenga E, Rayón C, Parody R, de la Serna J, et al. Differentiation syndrome in patients with acute promyelocytic leukemia treated with alltrans retinoic acid and anthracycline chemotherapy: characteristics, outcome, and prognostic factors. Blood. 2009 Jan 22; 113(4):775-83.

40 Cicconi L, Platzbecker U, Avvisati G, Paoloni F, Thiede C, Vignetti M, et al. Long-term results of all-trans retinoic acid and arsenic trioxide in non-high-risk acute promyelocytic leukemia: update of the APL0406 Italian-German randomized trial. Leukemia. 2020 Mar; 34(3):914-8. 
41 Abaza Y, Kantarjian H, Garcia-Manero G, Estey E, Borthakur G, Jabbour E, et al. Longterm outcome of acute promyelocytic leukemia treated with all-trans-retinoic acid, arsenic trioxide, and gemtuzumab. Blood. 2017 Mar 9;129(10):1275-83.

42 Cicconi L, Divona M, Ciardi C, Ottone T, Ferrantini A, Lavorgna S, et al. PML-RAR $\alpha$ kinetics and impact of FLT3-ITD mutations in newly diagnosed acute promyelocytic leukaemia treated with ATRA and ATO or ATRA and chemotherapy. Leukemia. 2016 Oct; 30(10):1987-92.

43 Burnett AK, Russell NH, Hills RK, Bowen D, Kell J, Knapper S, et al. Arsenic trioxide and all-trans retinoic acid treatment for acute promyelocytic leukaemia in all risk groups (AML17): results of a randomised, controlled, phase 3 trial. Lancet Oncol. 2015 Oct;16(13): 1295-305.

44 Adès L, Thomas X, Bresler AG, Raffoux E, Spertini O, Vey N, et al. Arsenic trioxide is required in the treatment of newly diagnosed acute promyelocytic leukemia. Analysis of a randomized trial (APL 2006) by the French Belgian Swiss APL group. Haematologica. 2018 Dec;103(12):2033-9.

45 Zhang X, Zhang H, Chen L, Wang M, Xi J, Liu $\mathrm{X}$, et al. Arsenic trioxide and all-trans retinoic acid (ATRA) treatment for acute promyelocytic leukemia in all risk groups: study protocol for a randomized controlled trial. Trials. 2018 Sep 5;19(1):476.

46 Efficace F, Mandelli F, Avvisati G, Cottone F, Ferrara F, Di Bona E, et al. Randomized phase III trial of retinoic acid and arsenic trioxide versus retinoic acid and chemotherapy in patients with acute promyelocytic leukemia: health-related quality-of-life outcomes. J Clin Oncol. 2014 Oct 20;32(30):3406-12.

47 Efficace F, Breccia M, Avvisati G, Cottone F, Intermesoli T, Borlenghi E, et al. Health-related quality of life, symptom burden, and comorbidity in long-term survivors of acute promyelocytic leukemia. Leukemia. 2019 Jul; 33(7):1598-607.

48 Kutny MA, Alonzo TA, Gerbing RB, Wang YC, Raimondi SC, Hirsch BA, et al. Arsenic trioxide consolidation allows anthracycline dose reduction for pediatric patients with acute promyelocytic leukemia: report from the children's oncology group phase III historically controlled trial AAML0631. J Clin Oncol. 2017 Sep 10;35(26):3021-9.

49 Creutzig U, Dworzak MN, Bochennek K, Faber J, Flotho C, Graf N, et al. First experience of the AML-Berlin-Frankfurt-Münster group in pediatric patients with standard-risk acute promyelocytic leukemia treated with arsenic trioxide and all-trans retinoid acid. Pediatr Blood Cancer. 2017 Aug;64(8):e26461.
50 Au WY, Chim CS, Lie AK, Liang R, Kwong $\mathrm{YL}, \mathrm{Zecca} \mathrm{M}$, et al. Combined arsenic trioxide and all-trans retinoic acid treatment for acute promyelocytic leukaemia recurring from previous relapses successfully treated using arsenic trioxide. Br J Haematol. 2019 Apr;117(1): $130-2$.

51 Coombs CC, DeAngelis LM, Feusner JH, Rowe JM, Tallman MS. Pseudotumor cerebri in acute promyelocytic leukemia patients on intergroup protocol 0129: clinical description and recommendations for new diagnostic criteria. Clin Lymphoma Myeloma Leuk. 2016 Mar;16(3):146-51.

52 Kayser S, Rahmé R, Martínez-Cuadrón D, Ghiaur G, Thomas X, Sobas M, et al. Outcome of older ( $\geq 70$ years) APL patients frontline treated with or without arsenic trioxide: an International Collaborative Study. Leukemia. $2020 \mathrm{Feb} 19$.

53 Lehmann S, Deneberg S, Antunovic P, Rangert-Derolf $\AA$, Garelius H, Lazarevic V, et al. Early death rates remain high in high-risk APL: update from the Swedish Acute Leukemia Registry 1997-2013. Leukemia. 2017 Jun; 31(6):1457-9.

54 Rosati S, Breccia M, Gurnari C, Carmosino I, Scalzulli E, Montefusco E, et al. Acute promyelocytic leukemia (APL) in very elderly patients: real-life behind protocols. Blood. 2019; 134(Suppl 1):3845.[Check Journal Title]

55 Zhu HH, Hu J, Lo-Coco F, Jin J. The simpler, the better: oral arsenic for acute promyelocytic leukemia. Blood. 2019 Aug 15;134(7):597605.

56 Qian LZY. The Cooperation Group of Phase II Clinical Trial of Compound Huangdai Tablet. Phase II clinical trial of compound Huangdai tablet in newly diagnosed acute promyelocytic leukemia. Chin J Hematol. 2006;27(12): 801-4.

57 Wang L, Zhou G, Liu P, Song J, Liang Y, Yan $X$, et al. Dissection of mechanisms of Chinese medicinal formula Realgar-Indigo naturalis as an effective treatment for promyelocytic leukemia. Proc Natl Acad Sci U S A. 2008 Mar 25;105(12):4826-31.

58 Zhu HH, Wu DP, Du X, Zhang X, Liu L, Ma $\mathrm{J}$, et al. Oral arsenic plus retinoic acid versus intravenous arsenic plus retinoic acid for non-high-risk acute promyelocytic leukaemia: a non-inferiority, randomised phase 3 trial. Lancet Oncol. 2018 Jul;19(7):871-9.

59 Zhu HH, Huang XJ. Oral arsenic and retinoic acid for non-high-risk acute promyelocytic leukemia. N Engl J Med. 2014 Dec 4;371(23): 2239-41.

60 Zhu HH, Liu YR, Jia JS, Qin YZ, Zhao XS, Lai YY. Oral arsenic and all-trans retinoic acid for high-risk acute promyelocytic leukemia. Blood. 2018 Jun 28;131(26):2987-9.
61 Jiang H, Liang GW, Huang XJ, Jiang Q, Han S, Shi LW, et al. Reduced medical costs and hospital days when using oral arsenic plus ATRA as the first-line treatment of acute promyelocytic leukemia. Leuk Res. 2015 Dec; 39(12):1319-24.

62 Chen Z, Guidez F, Rousselot P, Agadir A, Chen SJ, Wang ZY, et al. PLZF-RAR alpha fusion proteins generated from the variant $\mathrm{t}(11 ; 17)(\mathrm{q} 23 ; \mathrm{q} 21)$ translocation in acute promyelocytic leukemia inhibit ligand-dependent transactivation of wild-type retinoic acid receptors. Proc Natl Acad Sci U S A. 1994 Feb 1;91(3):1178-82.

63 Ciangola G, Gurnari C, Paterno G, Mirabile M, Angelini M, Lavorgna S, et al. STAT5bRARa-positive acute myeloid leukemia: diagnostic and therapeutic challenges of a rare AML subtype. Leuk Res. 2019 Mar;78:21-3.

64 Noguera NI, Catalano G, Banella C, Divona M, Faraoni I, Ottone T, et al. Acute promyelocytic leukemia: update on the mechanisms of leukemogenesis, resistance and on innovative treatment strategies. Cancers. 2019 Oct 18; 11(10):1591.

65 Goto E, Tomita A, Hayakawa F, Atsumi A, Kiyoi H, Naoe T. Missense mutations in PML-RARA are critical for the lack of responsiveness to arsenic trioxide treatment. Blood. 2011 Aug 11;118(6):1600-9.

66 Zhu HH, Qin YZ, Huang XJ. Resistance to arsenic therapy in acute promyelocytic leukemia. N Engl J Med. 2014 May 8;370(19):18646.

67 Lehmann-Che J, Bally C, de Thé H. Resistance to therapy in acute promyelocytic leukemia. N Engl J Med. 2014 Sep 18;371(12):1170-2.

68 Tomita A, Kiyoi H, Naoe T. Mechanisms of action and resistance to all-trans retinoic acid (ATRA) and arsenic trioxide (As2O3) in acute promyelocytic leukemia. Int J Hematol. 2013 Jun;97(6):717-25.

69 Iaccarino L, Ottone T, Divona M, Cicconi L, Cairoli R, Voso MT, et al. Mutations affecting both the rearranged and the unrearranged PML alleles in refractory acute promyelocytic leukaemia. Br J Haematol. 2016 Mar;172(6): 909-13.

70 Iaccarino L, Ottone T, Alfonso V, Cicconi L, Divona M, Lavorgna S, et al. Mutational landscape of patients with acute promyelocytic leukemia at diagnosis and relapse. Am J Hematol. 2019 Oct;94(10):1091-7.

71 Madan V, Shyamsunder P, Han L, Mayakonda A, Nagata Y, Sundaresan J, et al. Comprehensive mutational analysis of primary and relapse acute promyelocytic leukemia. Leukemia. 2016 Aug;30(12):2430-81. 